13.4

\title{
Источник двухчастотного перестраиваемого СВЧ-излучения на основе гироклистрона в режиме биений с входным сигналом
}

\author{
(C) Р.М. Розенталь, Н.С. Гинзбург, И.В. Зотова, А.С. Сергеев \\ Институт прикладной физики РАН, Нижний Новгород, Россия \\ E-mail: rrz@ipfran.ru
}

Поступило в Редакцию 19 августа 2021г.

В окончательной редакции 16 сентября 2021 г.

Принято к публикации 17 сентября 2021 г.

\begin{abstract}
Предложена новая схема источника двухчастотного излучения на основе усилителя гироклистронного типа, работающего в режиме самовозбуждения и управляемого входным сигналом. На примере мощного гироклистрона трехмиллиметрового диапазона показано, что при работе в режиме биений (т.е. вне полосы синхронизации) за счет варьирования частоты и уровня мощности входного сигнала можно реализовать непрерывную независимую перестройку расстояния между спектральными компонентами в диапазоне $10-150 \mathrm{MHz}$ и соотношения их амплитуд в диапазоне от -10 до $0 \mathrm{~dB}$.
\end{abstract}

Ключевые слова: гироклистрон, режим биений, многочастотный нагрев плазмы.

DOI: 10.21883/PJTF.2021.24.51798.18997

Успешные эксперименты по созданию источников многозарядных ионов на основе электронноциклотронного нагрева плазмы двухчастотным излучением сантиметрового диапазона $[1,2]$ имеют естественное продолжение и развитие при использовании более коротковолнового излучения, что позволяет поднять плотность плазмы и соответственно интенсивность ионных пучков. В традиционном одночастном варианте это достигается при использовании гироприборов миллиметрового диапазона [3]. Естественно, что двухчастотные колебания могут быть получены в комплексе, состоящем из двух гиротронов, работающих на общую нагрузку. Однако такое решение является достаточно дорогостоящим. Кроме того, весьма ограничена возможность взаимной перестройки частоты, а также управления амплитудой спектральных компонент, что требуется для решения задачи подавления плазменных неустойчивостей [4].

Значительно более технологичной представляется возможность получения многочастной генерации в одном гироприборе. В частности, в работах [5,6] были экспериментально реализованы мощные многочастотные источники К- и Ка-диапазонов на основе гирорезонансных ламп обратной волны. Однако определенным недостатком в этом случае также является отсутствие возможности независимой перестройки расстояния между спектральными компонентами и соотношения уровней излучения каждой из частотных компонент. В настоящей работе предлагается альтернативная схема источника многочастотного излучения с возможностью указанной перестройки на основе гироклистрона, работающего в режиме самовозбуждения выходного резонатора и управляемого входным сигналом.

Хорошо известно, что в синхронизированных генераторах при выходе за пределы полосы синхронизации воз- никают биения, частота которых непрерывно меняется при изменении частоты внешнего сигнала [7]. Данное явление экспериментально регистрировалось в СВЧгенераторах, таких как отражательный клистрон [8], виркатор [9], оротрон [10], а также в секционированных гирорезонансных усилителях, находящихся в режиме самовозбуждения, таких как гиротвистрон [11] и гироклистрон [12]. Выбор гироклистрона в качестве объекта исследования в данной работе обусловлен тем, что к настоящему времени указанные приборы экспериментально реализованы в миллиметровом диапазоне с уровнем выходной мощности в сотни киловатт $[13,14]$. Одновременно ведутся разработки подобных усилителей с мегаваттным уровнем мощности [15]. Таким образом, возможность создания на основе гироклистронов многочастотных источников излучения, управляемых внешним (входным) монохроматическим сигналом, представляется актуальной задачей.

Будем считать, что пространство взаимодействия гироклистрона длиной $z_{k}$ включает в себя входной и выходной резонаторы, возбуждаемые на моде $\mathrm{TE}_{m, q}$ при частоте, близкой к частоте отсечки, а также пространство дрейфа между ними. Исследование динамики гироклистрона будем проводить на основе самосогласованной системы уравнений с нефиксированной продольной структурой поля:

$$
\begin{gathered}
i \frac{\partial^{2} a}{\partial Z^{2}}+\frac{\partial a}{\partial \tau}+i \delta(Z) a=\frac{I_{0}}{2 \pi} \int_{0}^{2 \pi} p d \theta_{0}, \\
\frac{\partial p}{\partial Z}+\frac{g^{2}}{4} \frac{\partial p}{\partial \tau}+i p\left(\Delta-1+|p|^{2}\right)=-a,
\end{gathered}
$$

где $a=e A J_{m-s}\left(R_{0} \omega_{c} / c\right) / m c \bar{\omega}_{c} \gamma_{0} \beta_{\perp 0}^{3}, \quad \tau=\bar{\omega}_{c} t \beta_{\perp 0}^{4} / 8 \beta_{\| 0}^{2}$, $Z=\beta_{\perp 0}^{2} \bar{\omega}_{c} z / 2 \beta_{\| 0} c, \quad p=\left(p_{x}+i p_{y}\right) e^{-i \bar{\omega}_{c} t+i(m-1) \varphi} / p_{\perp 0}-$ 


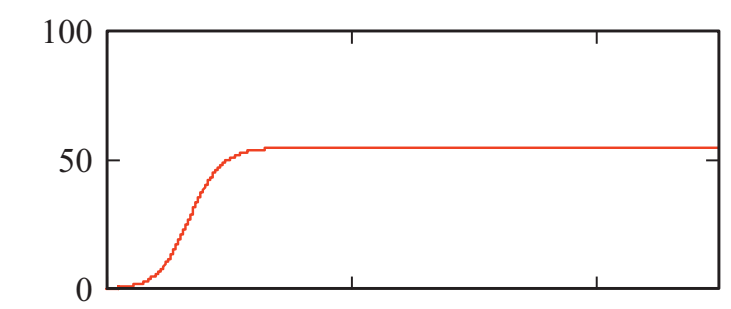

$a$
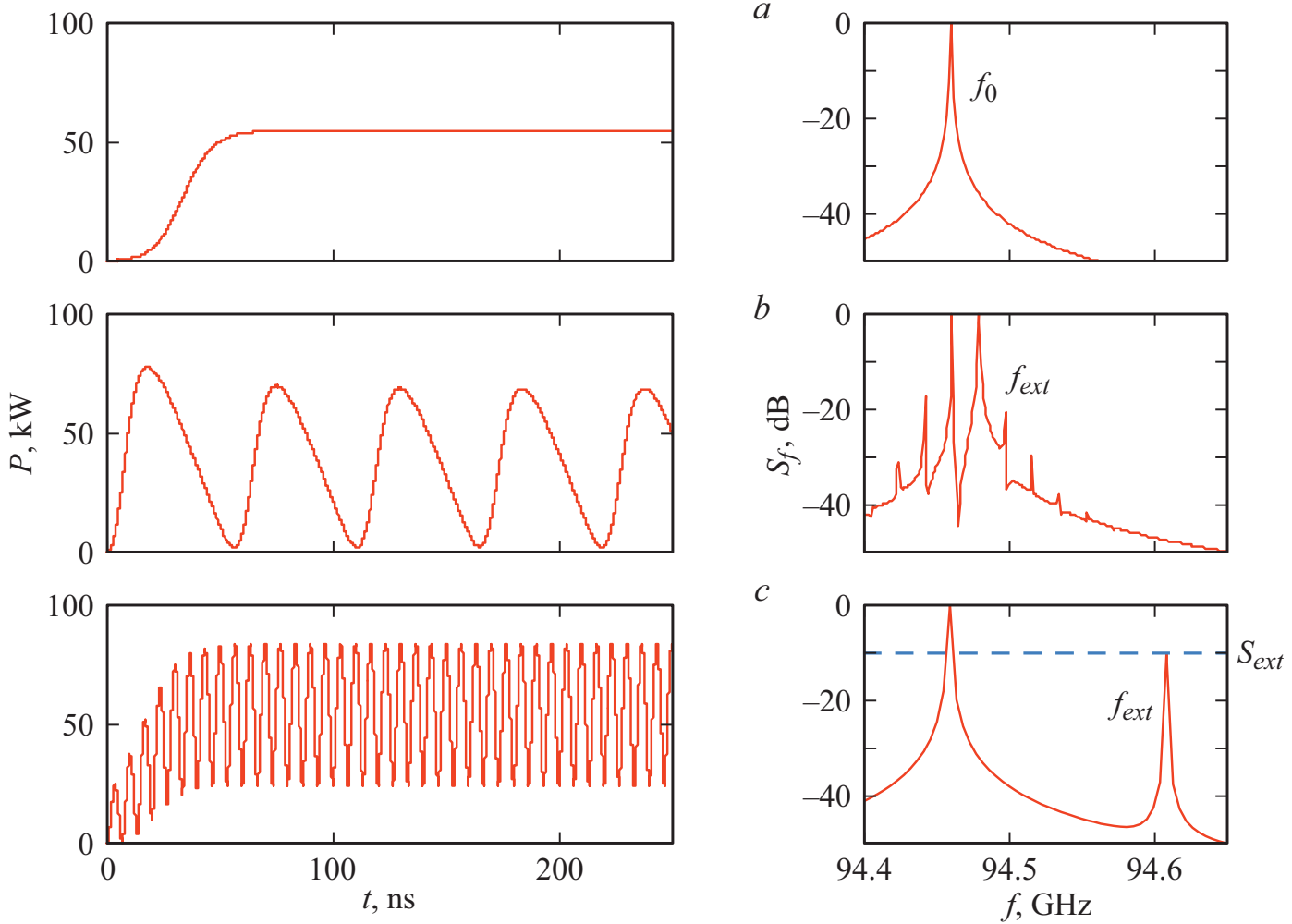

Рис. 1. Огибающая выходного сигнала и спектр излучения гироклистрона. $a-$ в режиме самовозбуждения; $b-$ в режиме биений при подаче внешнего сигнала относительно малой мощности $P_{e x t} / P_{0}=0.2 \%, f_{e x t}=94.48 \mathrm{GHz} ; c-$ в режиме биений при подаче внешнего сигнала относительно большой мощности $P_{\text {ext }} / P_{0}=2 \%, f_{\text {ext }}=94.61 \mathrm{GHz}$.

нормированные амплитуда поля возбуждаемой моды, время, продольная координата и поперечный импульс,

$$
I_{0}=16 \frac{e I_{b}}{m c^{3}} \frac{\beta_{\| 0}}{\beta_{\perp 0}^{6} \gamma_{0}} \frac{J_{m-1}^{2}\left(R_{0} \bar{\omega}_{c} / c\right)}{\left(v_{q}^{2}-m^{2}\right) J_{m}^{2}\left(v_{q}\right)}
$$

- параметр тока, $I_{b}$ - ток электронного пучка, $J_{m}$ функция Бесселя, $v_{q}-q$-й корень уравнения $J_{m}^{\prime}(v)=0$, $R_{0}$ - радиус инжекции винтового электронного пучка, $g=\beta_{\perp 0} / \beta_{\| 0}-$ питч-фактор на входе в пространство взаимодействия, $V_{\perp 0}=\beta_{\perp 0} c$ и $V_{\| 0}=\beta_{\| 0} c-$ начальные значения поперечной и продольной скорости электронов, $\Delta=2\left(\bar{\omega}_{c}-\omega_{H}\right) / \bar{\omega}_{c} \beta_{\perp 0}^{2}-$ параметр расстройки между критической частотой рабочей моды $\bar{\omega}_{c}$ в выходном сечении электродинамической системы и невозмущенным значением гирочастоты $\omega_{H}$. Функция $\delta(Z)=8 \beta_{\| 0}^{2}\left(\bar{\omega}_{c}-\omega_{c}(Z)\right) / \beta_{\perp 0}^{4} \bar{\omega}_{c} \quad$ описывает профиль электродинамической системы, $\omega_{c}(Z)=c v_{q} / r(Z)$, $r(Z)$ - зависимость радиуса от продольной координаты. На входе в пространство взаимодействия электроны равномерно распределены по фазам циклотронного вращения, так что $p(Z=0)=\exp \left(i \theta_{0}\right), \theta_{0} \in[0,2 \pi)$. На выходе системы в сечении $Z=L=\beta_{\perp 0}^{2} \bar{\omega}_{c} z_{k} / 2 \beta_{\| 0} c$ ставится излучательное граничное условие [16]:

$$
a(L, \tau)+\frac{1}{\sqrt{\pi i}} \int_{0}^{\tau} \frac{1}{\sqrt{\tau-\tau^{\prime}}} \frac{\partial a\left(L, \tau^{\prime}\right)}{\partial Z} d \tau^{\prime}=0 .
$$

В сечении $Z=0$ используется модифицированное граничное условие с учетом поступления на вход начального сигнала $F$ :

$$
a(0, \tau)-\frac{1}{\sqrt{\pi i}} \int_{0}^{\tau} \frac{1}{\sqrt{\tau-\tau^{\prime}}} \frac{\partial a\left(0, \tau^{\prime}\right)}{\partial Z} d \tau^{\prime}=2 F(0, \tau) .
$$

Дальнейший анализ проводился с использованием параметров экспериментально реализованного двухрезонаторного гироклистрона трехмиллиметрового диапазона [14]. В моделировании полагалось, что винтовой электронный пучок с энергией $70 \mathrm{keV}$, током $15 \mathrm{~A}$ и питч-фактором 1.3 инжектируется в гироклистрон с рабочей модой $\mathrm{TE}_{0,2}$ в обоих резонаторах. Расстройка циклотронного резонанса подбиралась таким образом, чтобы выходной резонатор самовозбуждался с максимальной выходной мощностью $P_{0}=55 \mathrm{~kW}$ (рис. $1, a$ ). Частота излучения при этом составляла $f_{0}=94.46 \mathrm{GHz}$ (W-диапазон).

При исследовании влияния входного сигнала на процесс генерации относительный уровень его мощности $P_{\text {ext }} / P_{0}$ выбирался равным 0.2 и $2 \%$. Частота входного сигнала $f_{\text {ext }}$ последовательно увеличивалась начиная со значения $f_{0}$. Ширина области синхронизации составила около $10 \mathrm{MHz}$ для случая $P_{\text {ext }} / P_{0}=0.2 \%$ и около $50 \mathrm{MHz}$ для случая $P_{\text {ext }} / P_{0}=2 \%$ (рис. 2). При выходе за пределы области синхронизации генератор пе- 


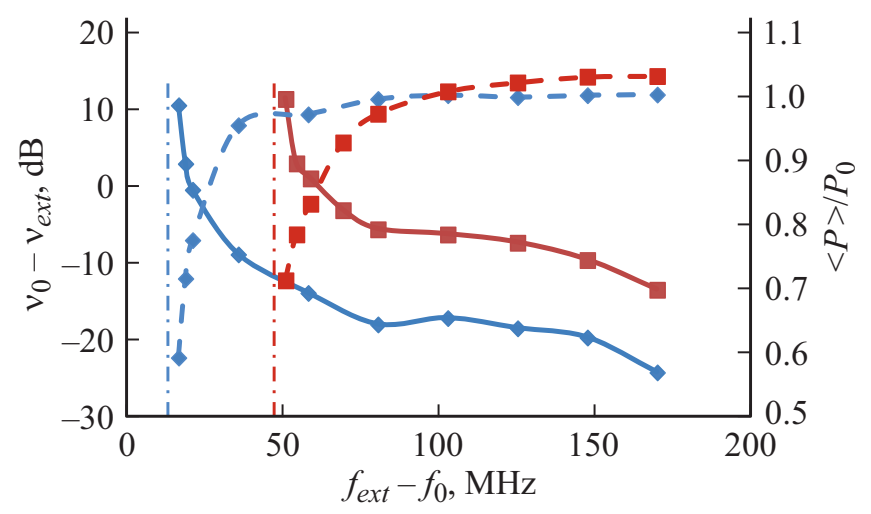

Рис. 2. Зависимость разности уровней основных спектральных компонент (сплошные линии) и относительной выходной мощности (штриховые линии) от разности частот собственной генерации и внешнего сигнала. $P_{\text {ext }} / P_{0}=0.2$ (ромбы) и $2 \%$ (квадраты). Вертикальными линиями обозначены соответствующие границы полосы синхронизации.

реходил в режим биений; при этом по мере увеличения частоты внешнего сигнала происходило непрерывное уменьшение амплитуды спектральной компоненты $S_{\text {ext }}$ на частоте внешнего сигнала относительно компоненты $S_{0}$, близкой к собственной частоте генерации. Согласно данным моделирования, при удалении от области захвата эффективность взаимодействия растет, а частота биений становится близкой к разности частот внешнего сигнала и собственной частоты генерации гироклистрона. Важно отметить, что подавляющая часть выходной мощности сосредоточена в двух соседних спектральных компонентах (рис. $1, b, c)$.

Расчеты показывают, что для случая $P_{\text {ext }} / P_{0}=0.2 \%$ интенсивности двух основных спектральных компонент сравниваются при отстройке частоты на величину около $20 \mathrm{MHz}$ (рис. $1, b$ ), а для случая $P_{\text {ext }} / P_{0}=2 \%$ - при отстройке на величину около $60 \mathrm{MHz}$. Средняя выходная мощность многочастотного излучения при этом составляет примерно $85 \%$ от величины $P_{0}$ (рис. 2).

Для задач подавления плазменных неустойчивостей при электронно-циклотронном нагреве плазмы в источниках многозарядных ионов достаточным является уровень дополнительной спектральной компоненты порядка $-10 \mathrm{~dB}$ [4]. Из рис. 2 видно, что для случая $P_{\text {ext }} / P_{0}=0.2 \%$ эта величина достигается при разности частот порядка $40 \mathrm{MHz}$, а для случая $P_{\text {ext }} / P_{0}=2 \%-$ порядка $150 \mathrm{MHz}$ (см. также рис. 1,c). Таким образом, варьируя частоту и мощность входного сигнала, можно осуществлять непрерывную перестройку расстояния между спектральными компонентами, сохраняя при этом неизменным отношение их амплитуд. Важно отметить, что при этом средняя по времени выходная мощность $\langle P\rangle$ практически достигает уровня мощности свободной генерации $P_{0}$ (рис. 2, штриховые линии).

Отметим, что по сравнению с вариантами автономных генераторов многочастотного излучения $[5,6]$ в предло- женной схеме требуется наличие источника внешнего сигнала. Для случая генерации излучения с частотой модуляции в несколько десятков мегагерц такими источниками могут быть лампы бегущей волны черенковского типа, непрерывная мощность генерации которых в рассматриваемом $\mathrm{W}$-диапазоне достигает $50 \mathrm{~W}$ [17]. В качестве более мощных источников могут использоваться гиротроны, для которых в последнее время предложены схемы, существенно увеличивающие диапазон частотной перестройки $[18,19]$.

Заметим в заключение, что еще одним практическим приложением излучения с глубокой модуляцией амплитуды может являться возбуждение электростатических ионно-циклотронных волн в плазме. Требуемые частоты модуляции при этом составляют величины порядка десятков мегагерц. В частности, в работе [20] для такого возбуждения предлагается использовать излучение двух мощных гиротронов, частоты которых должны быть разнесены на величину около $30 \mathrm{MHz}$. Однако, как уже отмечалось выше, подобное решение представляется дорогостоящим и ограниченным в возможностях перестройки частоты по сравнению с предложенным вариантом.

\section{Благодарности}

Авторы признательны И.В. Изотову и В.А. Скалыге за стимулирующие обсуждения в области создания многочастотных источников СВЧ-излучения.

\section{Финансирование работы}

Работа выполнена при поддержке Российского научного фонда (грант № 19-79-30071).

\section{Конфликт интересов}

Авторы заявляют, что у них нет конфликта интересов.

\section{Список литературы}

[1] V. Skalyga, I. Izotov, T. Kalvas, H. Koivisto, J. Komppula, R. Kronholm, J. Laulainen, D. Mansfeld, O. Tarvainen, Phys. Plasmas, 22 (8), 083509 (2015). DOI: 10.1063/1.4928428

[2] E. Naselli, D. Mascali, M. Mazzaglia, S. Biri, R. Rácz, J. Pálinkás, Z. Perduk, A. Galatá, G. Castro, L. Celona, S. Gammino, G. Torrisi, Plasma Sources Sci. Technol., 28 (8), 085021 (2019). DOI: 10.1088/1361-6595/ab32f9

[3] В.А. Скалыга, С.В. Голубев, И.В. Изотов, Р.Л. Лапин, С.В. Разин, А.В. Сидоров, Р.А. Шапошников, Прикладная физика, № 1, 17 (2019).

[4] A.G. Shalashov, E.D. Gospodchikov, I.V. Izotov, Plasma Phys. Control. Fusion, 62 (6), 065005 (2020). DOI: 10.1088/1361-6587/ab7f98

[5] Р.М. Розенталь, С.В. Самсонов, А.А. Богдашов, И.Г. Гачев, М.Ю. Глявин, Письма в ЖТФ, 47 (6), 11 (2021). DOI: 10.21883/PJTF.2021.06.50750.18510 
[6] Р.М. Розенталь, С.В. Самсонов, А.А. Богдашов, И.Г. Гачев, А.Н. Леонтьев, Н.С. Гинзбург, Письма в ЖТФ, 47 (19), 26 (2021). DOI: 10.21883/PJTF.2021.19.51509.18868

[7] R. Adler, Proc. IRE, 34 (6), 351 (1946).

DOI: $10.1109 / J R P R O C .1946 .229930$

[8] T.J. Buchanan, Proc. IRE, 40 (8), 958 (1952). DOI: $10.1109 / J R P R O C .1952 .274108$

[9] D. Price, H. Sze, D. Fittinghoff, J. Appl. Phys., 65 (12), 5185 (1989). DOI: $10.1063 / 1.343148$

[10] R.W. McMillan, D.M. Guillory, R.G. Hay, D.E. Wortman, H. Dropkin, J.M. Cotton, IEEE Trans. Microwave Theory Tech., 37 (11), 1828 (1989). DOI: 10.1109/22.41056

[11] J. Rodgers, H. Guo, V.L. Granatstein, S.H. Chen, G.S. Nusinovich, M. Walter, J. Zhao, IEEE Trans. Plasma Sci., 27 (2), 412 (1999). DOI: 10.1109/27.772268

[12] Ю.В. Новожилова, Н.М. Рыскин, Н.И. Зайцев, Ю.М. Гузнов, в сб Тр. 24-й Межждународной Крымской конференции „СВЧ-техника и телекоммуникационные технологии“ (КрыМиКо'2014) (Севастополь, 2014), с. 847.

[13] И.И. Антаков, И.Г. Гачев, Е.В. Засыпкин, Изв. вузов. Радиофизика, 54 (3), 185 (2011).

[14] Е.В. Засыпкин, И.Г. Гачев, И.И. Антаков, Изв. вузов. Радиофизика, 55 (5), 341 (2012).

[15] L.J.R. Nix, L. Zhang, W. He, C.R. Donaldson, K. Ronald, A.W. Cross, C.G. Whyte, Phys. Plasmas, 27 (5), 053101 (2020). DOI: $10.1063 / 1.5144590$

[16] N.S. Ginzburg, G.S. Nusinovich, N.A. Zavolsky, Int. J. Electron., 61 (6), 881 (1986). DOI: $10.1080 / 00207218608920927$

[17] C. Paoloni, D. Gamzina, R. Letizia, Y. Zheng, N.C. Luhmann, Jr., J. Electromagn. Waves Appl., 35 (5), 567 (2021). DOI: 10.1080/09205071.2020.1848643

[18] М.Ю. Глявин, А.Э. Федотов, И.В. Зотова, А.Г. Лучинин, М.Д. Проявин, Р.М. Розенталь, В.П. Тараканов, Изв. вузов. Радиофизика, 61 (11), 895 (2018).

[19] Р.М. Розенталь, И.В. Зотова, М.Ю. Глявин, А.Э. Федотов, Н.С. Гинзбург, А.С. Сергеев, В.П. Тараканов, Изв. вузов. Радиофизика, 63 (5-6), 403 (2020).

[20] H.P. Laqua, D. Moseev, P. Helander, P. Aleynikov, S. Marsen, N.B. Maruschenko, T. Stange, Nucl. Fusion, 58 (10), 104003 (2018). DOI: 10.1088/1741-4326/aad754 